\title{
Layered structures in extended dust clouds under microgravity
}

Cite as: Phys. Plasmas 25, 083707 (2018); https://doi.org/10.1063/1.5046049

Submitted: 25 June 2018 . Accepted: 30 July 2018 . Published Online: 16 August 2018

Michael Himpel (D), Stefan Schütt (D), Wojciech J. Miloch (D), and André Melzer
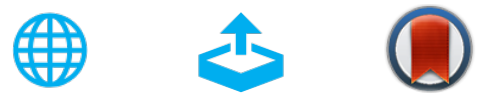

View Online

\section{ARTICLES YOU MAY BE INTERESTED IN}

Dust density waves in a dc flowing complex plasma with discharge polarity reversal Physics of Plasmas 25, 083705 (2018); https://doi.org/10.1063/1.5040417

Nonplanar dust-acoustic waves and chaotic motions in Thomas Fermi dusty plasmas Physics of Plasmas 25, 083706 (2018); https://doi.org/10.1063/1.5016893

A dust particle based technique to measure potential profiles in a plasma

Physics of Plasmas 25, 083711 (2018); https://doi.org/10.1063/1.5039429

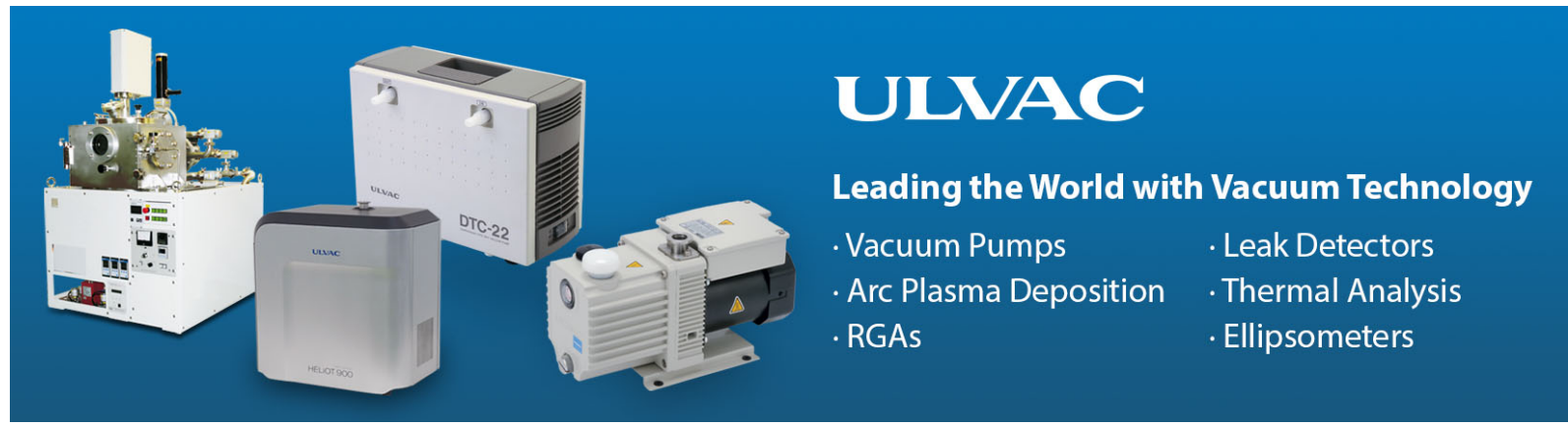




\title{
Layered structures in extended dust clouds under microgravity
}

\author{
Michael Himpel, ${ }^{1, a)}$ Stefan Schütt, ${ }^{1}$ Wojciech J. Miloch, ${ }^{2}$ and André Melzer ${ }^{1}$ \\ ${ }^{1}$ University Greifswald, 17489 Greifswald, Germany \\ ${ }^{2}$ Department of Physics, University of Oslo, 0316 Oslo, Norway
}

(Received 25 June 2018; accepted 30 July 2018; published online 16 August 2018)

\begin{abstract}
Three dimensional particle clouds in a plasma environment have been studied under microgravity conditions on parabolic flights. By using a stereoscopic 4-camera system, it was possible to reconstruct the three-dimensional particle motion in a selected volume. From this, a layered structure in the dust-cloud is revealed that is not observable with plain two-dimensional diagnostics. The spacing between the layers is found to be decisively larger than the interparticle distance inside the layer. This layered structure is observed in different situations, with and without waves. Further, it has been found that the position of the layers is stationary in the plasma even though the particles show an overall motion. The origin of the layer formation is discussed. Published by AIP Publishing. https://doi.org/10.1063/1.5046049
\end{abstract}

\section{INTRODUCTION}

A dusty or complex plasma is a plasma that contains microscopic "dust" grains. For laboratory measurements, manufactured micrometer-sized spheres with known diameters are generally used. When these particles are injected into a low-temperature plasma, they typically attain a negative charge due to electron and ion fluxes onto the particle surface. ${ }^{1,2}$ The charged particles interact mainly via screened Coulomb interaction and can show many interesting dynamic phenomena due to their collective behaviour. One such phenomenon is the dust-density wave (DDW) that is self-excited in the presence of a directed ion flow and a sufficiently high particle number density. ${ }^{3-5}$ Alternatively, structure formation in these extended dust clouds is of interest.

To investigate structures or waves with video cameras on the single-particle level, the particle size should be in the micrometer range to ensure sufficient brightness levels in the cameras. Unfortunately, the gravitational force is dominant for such particles in a plasma and results in problems to form three-dimensional particle clouds under laboratory conditions. To overcome sedimentation, experiments on the International Space Station, ${ }^{6,7}$ parabolic flights, ${ }^{8-12}$ and drop-tower experiments can be done. Our experiments have been performed under microgravity conditions on parabolic flights operated by Novespace in Bordeaux, France.

Three-dimensional particle diagnostics require elaborate diagnostics. Three-dimensional investigations on DDWs have been performed previously, but none of them was able to capture the full phase space information of all particles in the observation volume. Velocimetry-based methods do not deliver single particle trajectories but coarse-grained velocity fields. ${ }^{13,14}$ Three-dimensional stereoscopy with fluorescent tracer particles deliver only information of a small subset $(1 \%-5 \%)$ of the particles. ${ }^{15}$ Scanning microscopy is able to recover the three-dimensional structure of dust-clouds, but the temporal resolution is not able to cover dynamic phenomena adequately. ${ }^{7,16,17}$

\footnotetext{
${ }^{\text {a)} E l e c t r o n i c ~ m a i l: ~ h i m p e l @ p h y s i k . u n i-g r e i f s w a l d . d e ~}$
}

In this paper, we track the three-dimensional motion of most particles in the observation volume for many consecutive frames. For this purpose, we adopt the novel image analysis technique "Shake-the-box"18,19 that has been successfully applied in fluid dynamics investigations before. The temporal resolution of modern cameras is sufficient to fully resolve the dust motion even in fast phenomena like waves. In the following, the analysis of the threedimensional structure of dust clouds under microgravity will be presented. We study different systems with wave activity or pronounced string formation.

\section{SETUP AND ALGORITHM}

\section{A. Dusty plasmas under microgravity}

Figure 1(a) shows the IMPF-K2 plasma chamber used to produce our dusty plasma. ${ }^{20}$ It features a capacitively coupled argon plasma driven at a frequency of $13.56 \mathrm{MHz}$. The plasma power is typically varied between $P=2-4 \mathrm{~W}$ resulting in a voltage at the disk-shaped electrodes [see Fig. 1(b)] of $V_{\mathrm{pp}}=70-100 \mathrm{~V}$. The argon pressure is typically $p_{\mathrm{Ar}}=15-30 \mathrm{~Pa}$. The monodisperse microparticles are made from melamine-formaldehyde and have a diameter of $7.01 \mu \mathrm{m}$. The dust particles are injected by electromagnetically driven dispensers. During a parabolic flight, microgravity conditions are realized for about $20 \mathrm{~s}$ in each of the 31

(a)

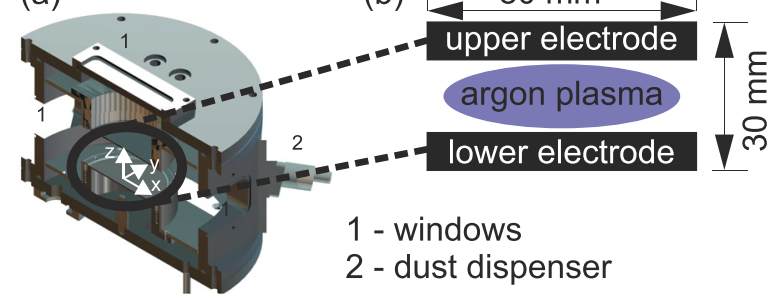

FIG. 1. (a) IMPF-K2 plasma chamber with several windows (1) and dustdispensers (2) to inject the microparticles. (b) The electrodes with $80 \mathrm{~mm}$ diameter are powered in push-pull mode and have a gap of approximately $30 \mathrm{~mm}$. 
parabolas per flight day. The time it takes for the dust cloud to settle and reach a stable structure is about 5-10 s. Even if the residual gravitational acceleration is small, it has noticeable erratic effects on the dust dynamics. Hence the analysis of the data is limited to sequences of only a few seconds where the residual gravity influence is small.

\section{B. Stereoscopic diagnostic}

The diagnostic setup used to investigate the motion of the particles consists of 4 cameras with monochrome CMOS sensors of 5.1 megapixel resolution. The cameras are equipped with $f=100 \mathrm{~mm}$ macro-lenses. This results in an observable field-of-view of about $16 \times 13 \mathrm{~mm}^{2}$ at the illumination plane. For our studies, a relatively wide laser sheet is used and the 3D motion of the particles within this wide laser sheet is studied. For this purpose, an illumination laser with a maximum cw-power of $1.5 \mathrm{~W}$ is transformed into a light sheet that has a thickness of about $2 \mathrm{~mm}$ which matches the depth-of-field of the optical system. The geometry of the setup is sketched in Fig. 2.

The cameras are synchronized by an external trigger signal. By selecting a restricted pixel region of the camera sensor a frame rate of up to $500 \mathrm{fps}$ can be realized to achieve a sufficient temporal resolution. Additionally, camera-internal $2 \times 2$ pixel-binning has been activated to maintain a sufficiently short exposure time and still have the desired contrast of the imaged particles. The resulting data rate was about $250 \mathrm{MB} / \mathrm{s}$ for each of the four cameras. The data of all four cameras has been recorded by a single PC with four USB3 interfaces. This relatively compact design of the setup and IT-infrastructure was necessary due to spatial restrictions in our parabolic flight experiment. As different structural states of the dust cloud have been investigated, different fields-ofview (FoV) have been chosen in different parabolas and dust clouds. In Fig. 3, the two FoVs used in this study are highlighted. Region "FoV 1" was used to record dust-density waves at $500 \mathrm{fps}$. In the second region, the nearly stationary cloud has been observed at $200 \mathrm{fps}$. In total, three measurements will be discussed in this paper. One measurement features a particle system with strong wave activity and large oscillation amplitudes at a neutral gas pressure of $p=15 \mathrm{~Pa}$. A second measurement was made at a pressure of $p=18 \mathrm{~Pa}$ resulting in less pronounced DDWs. The last measurement is taken from a dust cloud generated at $p=30 \mathrm{~Pa}$. The plasma

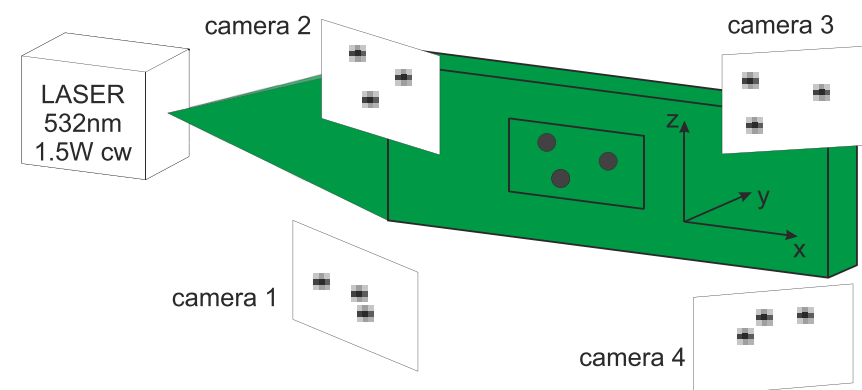

FIG. 2. Four cameras observe microparticles in a common volume inside the plasma. The scattered light of the particles on the sensor plane ideally yields a discretized Gaussian intensity profile. The depth of the reconstructed volume is defined by the $2 \mathrm{~mm}$ thick laser sheet.

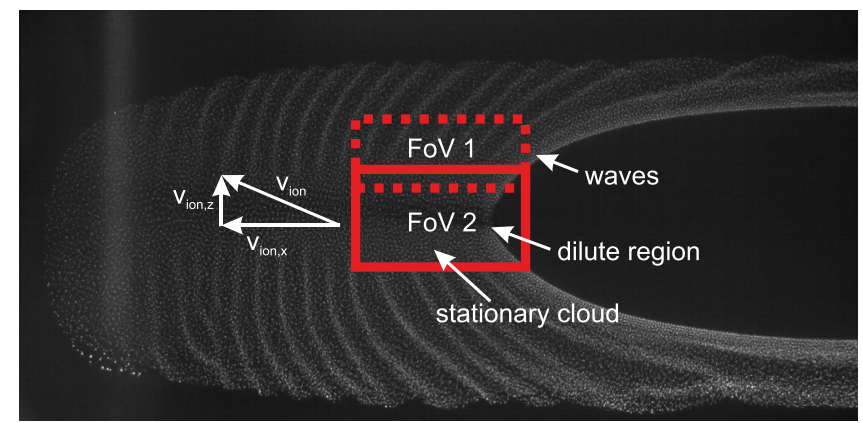

FIG. 3. Planar cross section through the dust cloud. The region labeled "FoV 1" was used to investigate dust-density waves (DDW) with a frame rate of $500 \mathrm{fps}$. In "FoV 2," the duct cloud was stable and featured strong coupling effects like a crystalline structure and lane formation.

power was $P=3 \mathrm{~W}$ for the first two measurements and $P=3.5 \mathrm{~W}$ for the third measurement.

\section{Three-dimensional reconstruction}

When a stereoscopic system with multiple cameras observe the same object under different angles, its threedimensional position can be determined from the twodimensional images. In our case we observe the dusty plasma using a stereoscopic setup with 4 cameras. First, the projective properties of the cameras must be known in form of a projection matrix $P_{\mathrm{i}}$ for each camera. We use our established calibration procedure in combination with a pinhole camera model. ${ }^{15,21}$ The projective pinhole camera model is sufficient here as considering radial and tangential distortion in our setup only results in deviations of $\approx 0.5 \mathrm{px}$ in the outermost image regions.

To determine the full phase space information of the particles, it is necessary to find the positions of the particles in real space and it is also necessary to link all particle observations in consecutive frames to trajectories. After this, the velocity and the acceleration of the particles can be found by numerical differentiation. In this experiment, all particles in the observation volume were aimed to be reconstructed. With a typical dust particle number density of $n_{\mathrm{d}} \approx 10^{11} \mathrm{~m}^{-3}$ about $N_{\mathrm{d}} \approx 10^{4}$ particles in the field-of-view are expected. This huge number of particles demands efficient calculations and carefully coded algorithms to keep the computation time as low as possible. Algorithms ${ }^{22-24}$ that perform well with a particle number $N_{\mathrm{d}} \ll 10^{4}$ cannot be applied in this case, as the time or/and the memory usage would be too exhaustive and many erroneous detections would impact the analysis quality.

\section{Shake-the-box applied in dusty plasmas}

Iterative particle reconstruction algorithms, such as the Shake-the-box-algorithm (STB), ${ }^{18}$ provide the capability to evaluate the trajectories of the indicated number of particles. For a complete and detailed description of STB, the reader is referred to Schanz, Gesemann, and Schröder, ${ }^{18}$ who have developed the algorithm to follow tracer particles in fluid systems. Here, only a brief introduction to the algorithm 
with emphasis on the modifications due to our imaging conditions will be given.

The general idea is that when some or most of the particle tracks over a few frames are known, one can use techniques, e.g., a Kalman filter, ${ }^{25,26}$ to predict the estimated location in 3D-space in the next frame. By "shaking" the predicted particle position, i.e., moving it along the 3D-axes, the position is iteratively matched to the observed particle projection in the camera images. The matching criterion is based on a comparison of the observed camera images and calculated artificial particle images. The following steps illustrate these main tasks:

\section{Finding initial tracks}

To start with the iteration procedure, it is necessary to have a sufficient amount of trajectories in the first frames. This can be done using computationally expensive algorithms. For our measurements, we use either direct triangulation $^{27}$ or tomographic reconstruction ${ }^{28}$ to find 3D particle positions in the first 3 frames. All particles in the first frame are associated with a new trajectory for each of them and their Kalman filter is initialized with that position. In the next frame it is tried to continue the trajectories using a nearest neighbor approach with a search radius of typically $0.05 \mathrm{~mm}$. If a suitable particle successor is found, the trajectory is continued with this position and the Kalman filter state is updated accordingly. In following frame(s), the prediction of the Kalman filter is used as the expected particle position and a nearest neighbor search for measured positions is again employed around this position to continue the particle trajectories.

\section{Predict new positions}

After three consecutive frames, the Kalman filter is already capable of yielding reasonable position predictions for the next frame. MATLAB's Kalman filter implementation can use a "constant velocity" or a "constant acceleration" model. We find that the constant acceleration model is better suited to particles in dust-density waves, where the particles are subject to an accelerated motion most of the time. The constant velocity model is better suited for a more steady dust cloud. At this point, it is crucial to ensure that the temporal resolution must be sufficient to resolve the observed motion. Undersampling may cause the predicted positions to deviate substantially from the true positions in the next frame. Higher frame rates decrease the prediction error but increase the computation time. The dust particle motion takes place on a time scale of $\approx 0.1 \mathrm{~s}$, so our chosen frame rates of $>200 \mathrm{fps}$ are able to capture the particle dynamics.

\section{Project current position to image}

When the projective properties of each camera are known, one can easily determine the image point $x_{\mathrm{p}, \mathrm{i}}$ of a particle $p$ in camera $i$ by $x_{\mathrm{p}, \mathrm{i}}=P_{\mathrm{i}} \cdot \vec{X}_{\mathrm{p}}$, where $\vec{X}_{\mathrm{p}}$ is the homogeneous position vector $[X, Y, Z, 1]^{\mathrm{T}}$ with $X, Y$ and $Z$ being the 3D-space coordinates and $T$ indicating the transposed form. For more details on this homogeneous representation of

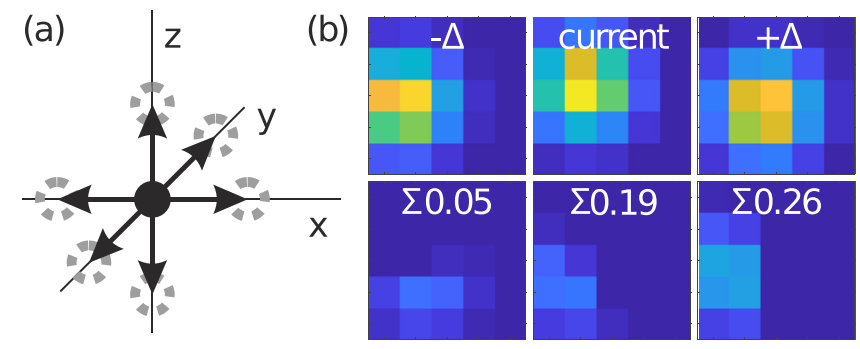

FIG. 4. (a) "Shaking" the particle means to shift the position by a given step size along the $x$-, $y$-, and $z$-axis. (b) Exemplary shift on one axis: The three top images indicate the projections of the current position (center) $X_{\mathrm{p}}$ and $X_{\mathrm{p}} \pm \Delta X$ (left and right). The bottom images show the residual $\sum\left(I_{\text {meas }}-I_{\text {projected }}\right)$ of the images.

vectors and matrices the reader is referred to textbooks on projective geometry. ${ }^{29}$ The particle positions $\vec{X}_{\mathrm{p}}$ are used to generate an artificial projected particle image for each camera. A particle in the computed image is modeled by a twodimensional Gaussian intensity profile in a $10 \times 10$ pixels window. The width of the Gaussian typically is a few pixels and must be adapted to the approximate particle size in each recorded image. Due to Mie scattering properties, even slight deviations in the viewing angle can lead to large deviations in the scattering intensity of micrometer-sized particles. ${ }^{30,31}$ Hence, the intensity of a particle projection is, in general, different in different cameras when working with dusty plasmas. So, in contrast to the original STB algorithm, the particle intensity is iteratively computed and tracked for each camera individually. Unfortunately, by losing the intensity as a camera-independent particle property it is more difficult to identify erroneous detections, as those often result in a higher particle intensity in one of the cameras.

\section{Shake particle until projection matches measurement}

Starting with the particle position $\vec{X}_{\mathrm{p}}$ predicted by the Kalman filter, the particle is moved by an amount $\Delta$ along the 3D axes ("shaking") as sketched by Fig. 4(a). Then, artificial image subsets are created that project the particle from the shake positions as shown in the upper images in Fig. 4(b). Finally, the residual between the artificial computed image and the measured image is computed as shown in the bottom row of Fig. 4(b). In the example from Fig. 4(b), the position $X-\Delta X$ will be used as the next current position to continue the iterative process, as its residual is the smallest one. The position has converged when the current position results in the smallest residual. It is promising to start with two coarse shaking iterations $\left(\Delta_{1}=15 \mu \mathrm{m}\right)$ followed by eight to ten fine shaking iterations $\left(\Delta_{2}=7 \mu \mathrm{m}\right)$. That way, it is possible to account for larger deviations of the actual particle position from the predicted position.

\section{Discontinue trajectories}

In the application of the algorithm to dusty plasmas, the following two conditions are used to discontinue a particle trajectory: Either the intensity of a particle falls below a given threshold in one of the cameras (since, e.g., there is no particle at the projected location in the recorded image), or 
the iterative shaking process does not converge during the maximum number of iterations allowed (typically eight to ten).

\section{Starting new trajectories}

After the shaking iterations, the predicted positions have been either confirmed and optimized or declined. For a second cycle, all confirmed particles are projected to build an artificial image which is then subtracted from the measured image. This residual image now contains mainly particles that have newly entered the FoV or particles whose trajectory has been discontinued prematurely. It is then processed again by triangulation or tomographic algorithms to find the positions $X_{\mathrm{p}}$ of the remaining visible particles. These particles then undergo a usual shaking optimization cycle. When necessary, in a third cycle the constraints for successful particle identification are somewhat loosened to find particles that are still not detected. Most, but not all, particle positions can be successfully reconstructed. Missing particles result mostly from not optimal image quality. The depthof-field was not perfectly adjusted or became misaligned during vibrations in the parabolic flight. Then, the cameras are not all focused on the exact same spatial volume and some detectable particles in one camera can give only very weak signals in another camera.

\section{Implementation}

The presented algorithm has been implemented in MATLAB coding language. The source-code and the license information are available for free and public download. ${ }^{32}$

\section{RESULTS}

\section{A. Reconstruction of layered structures}

The spatial accuracy of the reconstructed positions can, in general, be assumed to be approximately $\Delta_{2}$ which is $7 \mu \mathrm{m}$ in our case with a camera pixel corresponding approximately to $10 \mu \mathrm{m}$ in real space. For a statistical benchmark of the accuracy, particle positions from simulations of moving particles have been used to construct artificial camera images with approximately the same particle densities and velocities as in our measurements. These images have been analyzed with the STB algorithm and the resulting particle positions are compared with the input data. The resulting standard deviation of the reconstruction is smallest in $x$ - and $z$-direction $(\delta x=1.7 \mu \mathrm{m}, \delta z=2.2 \mu \mathrm{m})$ and somewhat larger in $y$-direction $(\delta y=2.8 \mu \mathrm{m})$. As the artificial benchmark images have been created using given camera projection matrices, the measurement is in perfect match with the calibration and the particle projections have a nice spherical shape with Gaussian intensity distribution. This results in the ability to reconstruct virtually all test particles. In the original publication, ${ }^{18}$ the authors reconstructed virtual particle distributions with a seeding density of up to $0.125 \mathrm{ppp}$ (particles per pixel). Our camera images have a mean particle density of about $0.01 \mathrm{ppp}$ (2D particle distance $\approx 7 \mathrm{px}$ ) and a maximum density of about $0.1 \mathrm{ppp}$ in the crest region of DDWs. Thus, the algorithm is capable of analyzing our measurements. In the presented actual measurements, it was possible to retrieve about $80 \%$ of the particle trajectories in a dust cloud with slow dynamics and about $50 \%$ of the particles in a dust cloud with strong particle motion. This results in an unprecedented amount of 3D trajectories.

In this paper, the data from three different measurements have been evaluated with the STB algorithm. All data sequences have a length of about 400 frames. The experiments done at neutral gas pressures of $15 \mathrm{~Pa}$ show strong self-excited waves. As increasing the neutral gas pressure leads to increased damping of the particle motion, in a second measurement at $18 \mathrm{~Pa}$ there is only weak wave activity. In a third measurement at $30 \mathrm{~Pa}$ the dust cloud is nearly stationary without any wave activity. The stationary cloud has been imaged with $200 \mathrm{fps}$ and the density waves with 500 fps. These frame rates ensure that the particle dynamics is fully resolved, and the traveled distance between two frames is well below the typical inter-particle distance. In Fig. 5, two data sets are shown with the $y$-coordinate in the upper image being color-coded. Figure 5(a) shows a small
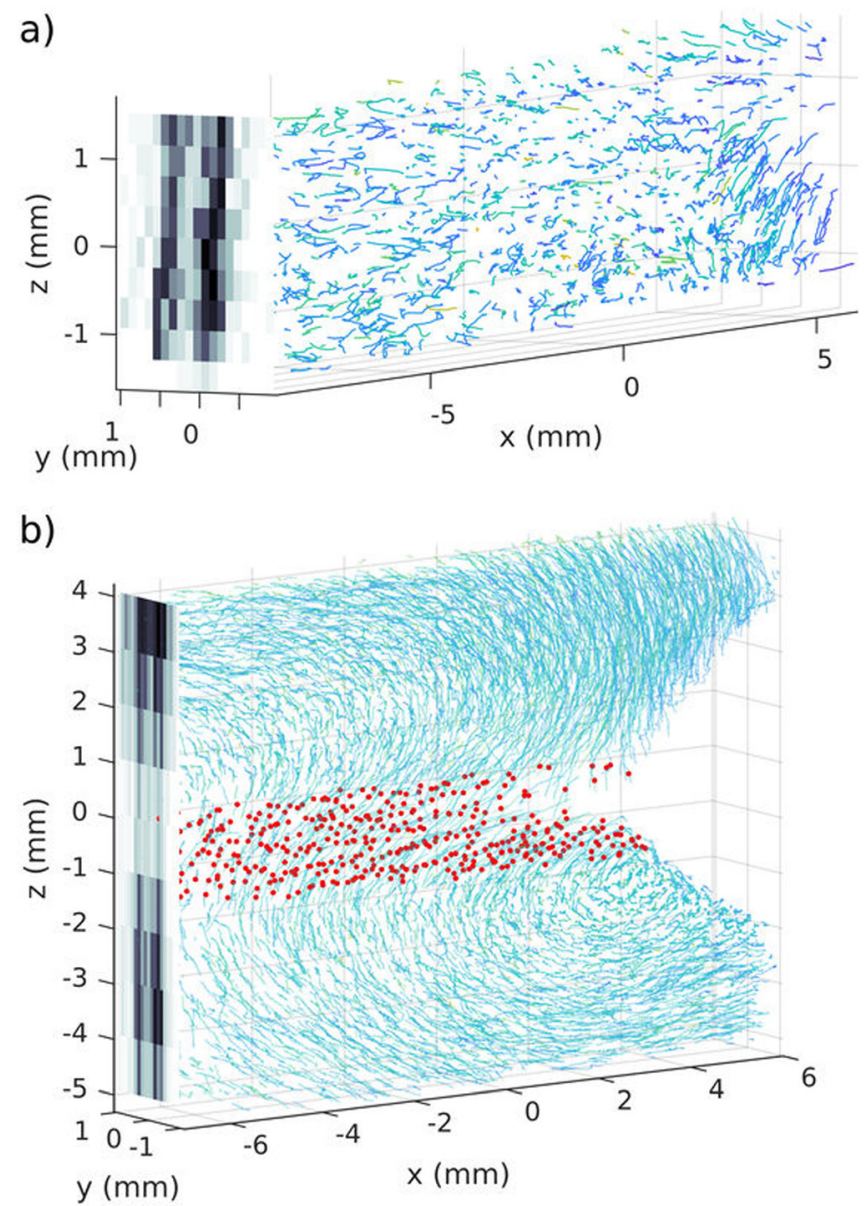

FIG. 5. Samples of reconstructed particle trajectories. The depth $y$ of the particles is color-coded in the upper image. (a) Particles in a dust-density wave. Mapping and binning the particle positions to the $y$-z-plane, one finds two vertical particle planes, one at $y \approx 0 \mathrm{~mm}$ and a second at $y \approx 0.5 \mathrm{~mm}$. (b) Particles in a nearly stationary dust cloud. The layers are less pronounced, and the density is generally lower in the midplane region around $z \approx-0.5 \mathrm{~mm}$. Particles belonging to the dilute midplane region are highlighted as red dots. 
fraction of particle trajectories forming a dust-density wave. About 1300 from 2600 visible particles per frame have been reconstructed. Figure 5(b) shows a measurement, where no self-excited waves have been present. Here, about 4000 out of 5000 visible particles are reconstructed in every frame. Additionally, the particle number density in the $y-z$-plane is shown by counting the particles and averaging over the $x$-coordinate. It shows a clear indication of a layered structure along the $y$-direction. In the more steady dust cloud, the same layer structure can be identified in the upper and lower regions. In the midplane region near $z=-1 \mathrm{~mm}$, the particle arrangement is very dilute. There, the particles are ordered preferably in strings. The large inter-string separation perpendicular to the $x$-axis results in a total reduced particle number density compared to the upper and lower regions of the dust cloud. The trajectories indicate that the dust cloud is not fully at rest. This may be caused by minor residual gravity or the cloud having not fully reached its equilibrium state yet. Additionally there is a slow vortex motion present, similar to the one observed in other dusty plasma experiments. $^{33,34}$

\section{B. Particle and layer separation}

To further analyze the structure of the different particle systems, the particle distribution in $y$-direction (averaging over the full range of the other coordinates) has been computed. In Fig. 6, density distributions of three measurements are shown, one with strong wave activity [see also Fig. 5(a)], one with weak wave activity, and one with the nearly stationary cloud. The density distribution shows two clear maxima, the steady cloud even three (or at least two humps and a shoulder). All datasets could be nicely fitted by a double or a triple Gaussian. From the fit to the data, the separation of the two peaks is $d=550 \mu \mathrm{m}$ and $d=534 \mu \mathrm{m}$ for the layer separation in the presence of strong and weak waves, respectively. The distances between the three peaks from the steady cloud data are $d=587 \mu \mathrm{m}$ and $d=469 \mu \mathrm{m}$. Hence, we find vertical layers of about $530 \mu \mathrm{m}$ separation in different parts of the cloud (see resp. FoV 1\&2 in Fig. 3) with and without wave activity.

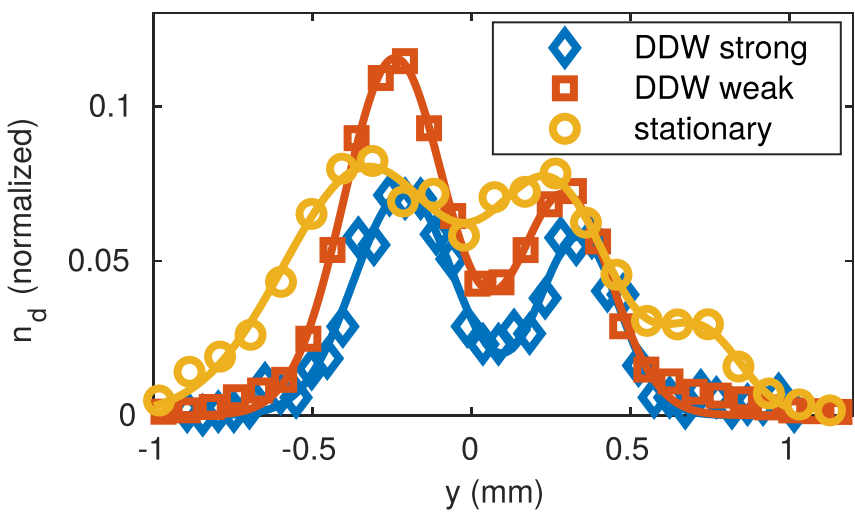

FIG. 6. Particle number density in the $y$-direction from 3 data sets and fitted Gaussians (solid lines). The strong and weak DDW data show two peaks with a separation of $d=550 \mu \mathrm{m}$ and $d=534 \mu \mathrm{m}$, respectively. The data from a steady dust cloud show three peaks with a separation of $d=587 \mu \mathrm{m}$ and $d=469 \mu \mathrm{m}$.
The particle separation inside the layers can be studied best in the stable cloud. There one can find regions with higher density and a dilute region $(z=-1.5 \mathrm{~mm}$ to $0 \mathrm{~mm})$, which has a non-isotropic structure. The dilute region shows by visual inspection clear formation of particle strings along the $x$-direction (see also Arp, Goree, and Piel ${ }^{35}$ ). These different structures have been analyzed by means of a pair correlation function $g_{\mathrm{ab}}(r) .^{36}$ It calculates the Euclidean distance $r$ in 3D-space for every particle $a$ to every other particle $b$ and counts the found particles in their respective distances. The first correlation (blue squares in Fig. 7) is computed from the upper dense region of the steady dust cloud [see Fig. 5(b), $z=1$ to $3 \mathrm{~mm}$ ], averaged over 100 frames. The peak of $g_{\mathrm{ab}}(r)$ at $d \approx 310 \mu \mathrm{m}$ can be identified with the mean nearest-neighbor distance of the particles inside the layer. To determine the particle distance in the dilute region with string formation $(z=-1.5$ to $0 \mathrm{~mm}$ ), the function $g_{\mathrm{ab}}(r)$ was, first, calculated with particles $b$ being located in a restricted cylindrical tube around particle $a$ with $\sqrt{\left(Y_{\mathrm{b}}-Y_{\mathrm{a}}\right)^{2}+\left(Z_{\mathrm{b}}-Z_{\mathrm{a}}\right)^{2}}<0.2 \mathrm{~mm}$ (see red circles). This way, the pair correlation in the $x$-direction, which is the dominant direction of the ion stream and string formation, can be investigated. The particle distance along the $x$-axis in this dilute region is found to be about $d \approx 240 \mu \mathrm{m}$ from one particle to its nearest neighbor in the same string. The second peak at $d \approx 500 \mu \mathrm{m}$ indicates the distance to the second neighbor in the same string. The clear maxima indicate that this region is relatively highly ordered and the particles in the strings are very close to each other. However, the stringto-string distance perpendicular to the $x$-axis (string axis), see yellow diamonds in Fig. 7, is not well defined. The correlation function shows a broad distribution with distances larger than at least $400 \mu \mathrm{m}$. This indicates that the arrangement of strings is quite unordered with an average density that is smaller than in the denser parts of the cloud.

The values for the separation of the dust particles are of the order of the Debye shielding length for the dust particles. In the given observation volumes, the Debye length $\lambda_{\text {D.eff }}$ can be estimated taking ion flows into account. ${ }^{37}$ By taking values

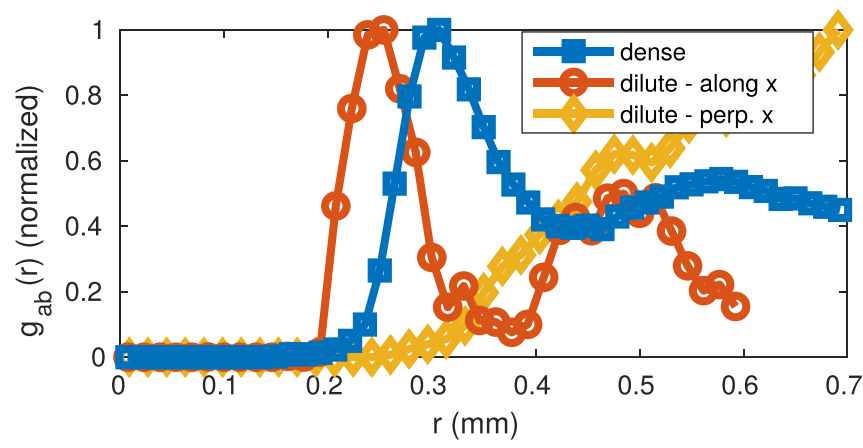

FIG. 7. The pair-correlation function $g_{\mathrm{ab}}(r)$ for particles in the nearly stationary dust cloud. The $g_{\mathrm{ab}}(r)$ from particles inside the dense (blue squares) and the dilute region is shown. The structure in the dilute region is analyzed along the $x$-axis, i.e., along the strings (red circles) and perpendicular to the $x$-axis (yellow diamonds). The blue and red graphs show two peaks which can be identified with the distance to the first and second nearest neighbors. The interparticle distance is found to be $d \approx 310 \mu \mathrm{m}$ in the dense region and $d_{x} \approx 240 \mu \mathrm{m}$ in the dilute region (along the $x$-axis) of the dust cloud. 
from a SIGLO2D simulation, ${ }^{33}$ one finds an electron temperature of $T_{\mathrm{e}}=100 \cdot T_{\mathrm{i}}=3 \mathrm{eV}$, an electron density $n_{\mathrm{e} 0}=n_{\mathrm{i} 0}$ $=10^{15} \mathrm{~m}^{-3}$, and a dust charge of $Z_{\mathrm{d}}=7000 \cdot e$. From that a screening length of $\lambda_{\mathrm{D} \text {,eff }} \approx 550 \mu \mathrm{m}$ in the dense regions with layer formation is obtained. In the lower density midplane region, one finds a screening length of $\lambda_{\mathrm{D} \text {, eff }} \approx 300 \mu \mathrm{m}$ due to a higher ion mobility. In summary, we find clear layer formation when the separation between the layers is decisively larger than the particle separation in the layer or within the strings.

\section{Layer stability}

As seen from the trajectories in Fig. 5, the measurement of the nearly stationary cloud features a continuous particle drift mainly in the negative $y$-direction of $v_{\mathrm{y}}=0.8 \mathrm{~mm} / \mathrm{s}$. To investigate the temporal behaviour of the layers, the particle number density distribution has been examined over 200 frames in smaller time steps of 10 frames. Figure 8 shows these 20 density distributions as thin curves and the distribution over the full frame range as a thicker curve. The time-resolved distributions show no significant deviation from the time-averaged distribution although the particles move about $0.8 \mathrm{~mm}$ during the covered period of time. Thus, the layered structures seem to be stationary and do not move with the particles. There seems to be a plasma-intrinsic origin of the layer formation. Another hint on a different source of energy responsible for the layers can be found in the velocity distribution. Figure 8 shows the root mean square of the $y$-velocity $v_{\mathrm{y}, \mathrm{rms}}$ as a function of $y$-position. Its peaks clearly coincide with the troughs of $n_{\mathrm{d}}$ and vice versa. This indicates that the particles have a higher rms velocity between the layers than in the dense regions within a layer.

\section{Influence of the laser}

One might argue that the illumination laser may cause the necessary potential to form the layers by either momentum transfer or a confining gradient force as used in optical tweezers. $^{38,39}$ Both cases are very unlikely to cause the observed particle structures for mainly two reasons: First, the laser has been broadened to $2 \mathrm{~mm}$ width for the

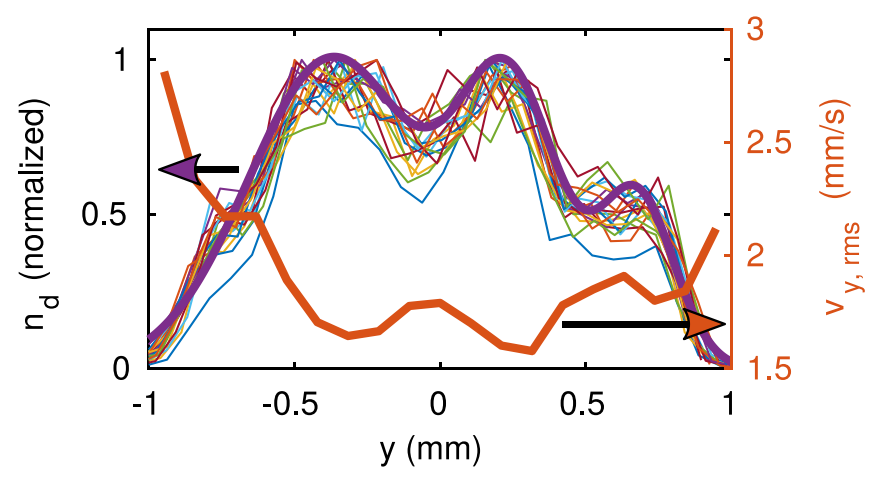

FIG. 8. (density) 20 thin lines show the particle number density $n_{\mathrm{d}}$ averaged over 10 frames each. Additionally, the bold curve shows a triple Gaussian fitted to the density averaged over all 200 frames. (velocity) The root-mean-square of the $y$-velocity is shown on the right axis and the bold red curve. Its peaks and troughs are inverse to the peaks and troughs of the density curve. stereoscopy. Hence, the laser power density is smaller than in all previous experiments. There, a laser influence on the particle motion has never been observed. Second, we have checked that the laser sheet has a (single) Gaussian intensity profile along the $y$-direction. This could -if strong enough - result in a single particle number density peak in the laser plane in contrast to the double or multi-layer structure that we observe.

\section{E. Ionization instability}

An effect that could cause the formation of the observed structures is the ionization instability. ${ }^{40}$ The main underlying process described by Morfill and Tsytovich ${ }^{40}$ is the intensified plasma generation in initial pertubations of the dust number density. This stronger plasma generation in the absence of dust, as known from the central void, results in an ion stream and thus an ion drag force onto the particles. This force supports the separation of stationary density pertubations as we have observed in our experiment. The expected typical scale length $\lambda \approx \lambda_{\mathrm{D}, \mathrm{i}}^{2} / d$ of the instability with $\lambda_{\mathrm{D}, \mathrm{i}}$ being the ion Debye length and $d$ being the particle diameter is $\lambda \approx 300 \mu \mathrm{m}$ with the parameters given in Sec. III B. The order of magnitude indeed reflects our measured layer separation distance. It is noteworthy that such an instability has been suspected to cause stationary dust density structures in another experiment. ${ }^{41}$ But, in contrast to our experiment, the structures discussed in Refs. 40 and 41 alternated in ion flow direction while our structures are oriented perpendicular to the ion flow.

\section{SUMMARY AND CONCLUSIONS}

In this paper, we presented measurements with a novel three-dimensional camera arrangement that has been set up to investigate three-dimensional effects of dusty plasmas under microgravity conditions. Using the Shake-the-box algorithm, it was possible to reconstruct a large amount of the particle trajectories with a high temporal resolution.

This three-dimensional insight revealed that the particles preferably organize themselves in layers with a distance of approximately the Debye shielding length. These layers have been found to exist in regions with dust-density waves as well as in dense stationary regions. Only the dilute low density midplane region of the dust cloud did not show the layered structure. There, the dominant particle structure was string formation. An analysis of a stationary cloud was done with the result that the layers do not co-move with the particles but are spatially stable. Analyzing the spatially resolved velocity of the particles in relation to the spatial particle distribution, we found a higher kinetic temperature of the particles between the layers. These observations hint to an ionization instability as the possible cause for the formation of the observed particle layers. Nevertheless, the same mechanism can be at work in our situation. Already a small perpendicular component of the ion radially outward ion flow (caused by the potential modulations of the instability) can lead to substantial perpendicular forces. This is 
because the perpendicular ion flow results in a perpendicular component of the already strong outward ion drag force.

\section{ACKNOWLEDGMENTS}

Financial support from the Deutsches Zentrum für Luftund Raumfahrt (DLR) under Project No. 50WM1139 is gratefully acknowledged.

${ }^{1}$ P. K. Shukla and A. A. Mamun, Introduction to Dusty Plasma Physics (Institute of Physics Publishing, Bristol, 2002).

${ }^{2}$ A. Piel, Plasma Physics (Springer, 2017).

${ }^{3}$ R. L. Merlino, J. Plasma Phys. 80, 773 (2014).

${ }^{4}$ N. Rao, P. Shukla, and M. Yu, Planet. Space Sci. 38, 543 (1990).

${ }^{5}$ F. Verheest, Waves in Dusty Space Plasmas (Springer, 2000).

${ }^{6}$ A. G. Khrapak, V. I. Molotkov, A. M. Lipaev, D. I. Zhukhovitskii, V. N. Naumkin, V. E. Fortov, O. F. Petrov, H. M. Thomas, S. A. Khrapak, P. Huber, A. Ivlev, and G. Morfill, Contrib. Plasma Phys. 56, 253 (2016).

${ }^{7}$ A. P. Nefedov, G. E. Morfill, V. E. Fortov, H. M. Thomas, H. Rothermel, T. Hagl, A. V. Ivlev, M. Zuzic, B. A. Klumov, A. M. Lipaev, V. I. Molotkov, O. F. Petrov, Y. P. Gidzenko, S. K. Krikalev, W. Shepherd, A. I. Ivanov, M. Roth, H. Binnenbruck, J. A. Goree, and Y. P. Semenov, New J. Phys. 5, 33 (2003).

${ }^{8}$ M. H. Thoma, H. Höfner, M. Kretschmer, S. Ratynskaia, G. E. Morfill, A. Usachev, A. Zobnin, O. Petrov, and V. Fortov, Microgravity-Sci. Technol. 18, 47 (2006).

${ }^{9}$ O. Arp, D. Caliebe, and A. Piel, Phys. Rev. E 83, 066404 (2011).

${ }^{10}$ K. O. Menzel, O. Arp, and A. Piel, Phys. Rev. E 83, 016402 (2011).

${ }^{11}$ B. Buttenschön, M. Himpel, and A. Melzer, New J. Phys. 13, 023042 (2011).

${ }^{12}$ H. M. Thomas, D. D. Goldbeck, T. Hagl, A. V. Ivlev, U. Konopka, G. E. Morfill, H. Rothermel, R. Stterlin, and M. Zuzic, Phys. Scr. 2001, 16; http://iopscience.iop.org/article/10.1238/Physica.Topical.089a00016.

${ }^{13}$ E. Thomas, J. D. Williams, and J. Silver, Phys. Plasmas 11, L37 (2004).

${ }^{14}$ J. D. Williams, Phys. Plasmas 18, 050702 (2011).

${ }^{15}$ M. Himpel, B. Buttenschön, and A. Melzer, Rev. Sci. Instrum. 82, 053706 (2011).

${ }^{16}$ M. Schwabe, M. Rubin-Zuzic, S. Zhdanov, A. V. Ivlev, H. M. Thomas, and G. E. Morfill, Phys. Rev. Lett. 102, 255005 (2009).

${ }^{17}$ K. O. Menzel, O. Arp, D. Caliebe, and A. Piel, IEEE Trans. Plasma Sci. 38, 838 (2010).
${ }^{18}$ D. Schanz, S. Gesemann, and A. Schröder, Exp. Fluids 57, 70 (2016).

${ }^{19}$ B. Wieneke, Meas. Sci. Technol. 24, 024008 (2013).

${ }^{20}$ M. Klindworth, O. Arp, and A. Piel, J. Phys. D: Appl. Phys. 39, 1095 (2006)

${ }^{21}$ Z. Zhang, IEEE Trans. Pattern Anal. Mach. Intell. 22, 1330-1334 (2000).

${ }^{22}$ Y. Akhmetbekov, V. Lozhkin, D. Markovich, and M. Tokarev, in 9th International Symposium on Particle Image Velocimetry-Piv (2011), Vol. 11, pp. 21-23.

${ }^{23}$ B. Wieneke, Exp. Fluids 45, 549 (2008).

${ }^{24}$ A. Melzer, M. Himpel, C. Killer, and M. Mulsow, J. Plasma Phys. 82, 615820102 (2016).

${ }^{25}$ R. E. Kalman, Trans. ASME-J. Basic Eng. 82, 35 (1960).

${ }^{26} \mathrm{G}$. Welch and G. Bishop, "An introduction to the kalman filter," Technical Report No. TR 95-041, Department Of Computer Science, University Of North Carolina at Chapel Hill, 2004.

${ }^{27}$ R. I. Hartley and P. Sturm, Comput. Vision Image Understanding 68, 146 (1997).

${ }^{28}$ G. E. Elsinga, F. Scarano, B. Wieneke, and B. W. van Oudheusden, Exp. Fluids 41, 933 (2006).

${ }^{29}$ R. Hartley and A. Zisserman, Multiple View Geometry in Computer Vision (Cambridge University Press, New York, NY, USA, 2000).

${ }^{30}$ C. Killer, M. Mulsow, and A. Melzer, Plasma Sources Sci. Technol. 24, 025029 (2015).

${ }^{31}$ C. G. Bohren and D. R. Huffman, Absorption and Scattering of Light by Small Particles (John Wiley and Sons, New York, 1983).

${ }^{32}$ M. Himpel, see https://physik.uni-greifswald.de/ag-melzer/forschung/ stereo-toolbox/ for stereo calibration and reconstruction toolbox.

${ }^{33}$ T. Bockwoldt, O. Arp, K. O. Menzel, and A. Piel, Phys. Plasmas 21, 103703 (2014).

${ }^{34}$ M. Schwabe, S. Zhdanov, C. Räth, D. B. Graves, H. M. Thomas, and G. E. Morfill, Phys. Rev. Lett. 112, 115002 (2014).

${ }^{35}$ O. Arp, J. Goree, and A. Piel, Phys. Rev. E 85, 046409 (2012).

${ }^{36}$ M. Zuzic, A. V. Ivlev, J. Goree, G. E. Morfill, H. M. Thomas, H. Rothermel, U. Konopka, R. Sütterlin, and D. D. Goldbeck, Phys. Rev. Lett. 85, 4064 (2000).

${ }^{37}$ I. H. Hutchinson, Plasma Phys. Controlled Fusion 48, 185 (2006).

${ }^{38}$ A. Ashkin, Phys. Rev. Lett. 24, 156 (1970).

${ }^{39}$ J. Schablinski, F. Wieben, and D. Block, Phys. Plasmas 22, 043703 (2015).

${ }^{40}$ G. Morfill and V. N. Tsytovich, Plasma Phys. Rep. 26, 682 (2000).

${ }^{41}$ J. R. Heinrich, S.-H. Kim, and R. L. Merlino, Phys. Rev. E 84, 026403 (2011). 